\title{
Electron spin resonance of Er-oxygen complexes in GaAs grown by metal organic chemical vapor deposition
}

\author{
T. Ishiyama, ${ }^{\text {a) }}$ E. Katayama, and K. Murakami \\ Institute of Materials Science, University of Tsukuba, 1-1-1 Tennoudai, Tsukuba, Ibaraki 305, Japan \\ K. Takahei ${ }^{\text {b) }}$ and A. Taguchi \\ NTT Basic Research Laboratories, 3-1 Morinosato-Wakamiya, Atsugi, Kanagawa 243-01, Japan
}

(Received 5 June 1998; accepted for publication 16 September 1998)

\begin{abstract}
We have performed electron spin resonance (ESR) measurements on Er-doped GaAs grown with oxygen codoping by metal organic chemical vapor deposition. An isotropic line (an effective $g$ value, $g=5.95$ ) which had been already reported was observed in samples without oxygen codoping. On the other hand, for samples with oxygen codoping other strong anisotropic ESR lines originated from four kinds of $\mathrm{Er}^{3+}\left(4 f^{11}\right)$ centers (A, B, C, and D) were newly observed in addition to the weaker isotropic line. The anisotropic $g$ tensors obtained by analyzing the angular dependence of the ESR lines indicate that $\mathrm{B}$ and $\mathrm{C}$ centers are of orthorhombic $C_{2 v}$ symmetry, A center has lower symmetry than orthorhombic symmetry, and D center is of trigonal $C_{3 i}$ symmetry. The ESR intensities of A, B, and C centers were approximately two orders of magnitude higher than that of the isotropic line with $g=5.95$. The ESR intensity of D center was one order of magnitude lower than those of A, B, and C. The Er concentration dependence of the relative ESR intensities of these centers was investigated, which indicates (i) the ESR intensities of A and D increase with increasing Er concentration, and (ii) those of $\mathrm{B}$ and $\mathrm{C}$ are saturated above the Er concentration [Er] $\geqslant 10^{18} \mathrm{~cm}^{-3}$. The ESR measurement under light illumination, as well as the Er concentration dependence, suggests that the $\mathrm{B}$ center with $C_{2 v}$ symmetry corresponds to the dominant $\mathrm{Er}$ luminescent center under host photoexcitation. () 1998 American Institute of Physics.
\end{abstract}

[S0021-8979(98)03724-4]

\section{INTRODUCTION}

Semiconductors doped with rare earth impurities exhibit sharp and temperature-insensitive photoluminescence (PL) and electroluminescence due to the intra- $4 f$-shell transition. These semiconductors have widely been studied because of their possible applications to new optical devices. In particular, Er-doped semiconductors such as GaAs and Si have attracted much attention, because the wavelength of the luminescence due to the ${ }^{4} I_{13 / 2} \rightarrow{ }^{4} I_{15 / 2}$ intra- $4 f$-shell transition of $\mathrm{Er}^{3+}\left(4 f^{11}\right)$ is in the $1.5 \mu \mathrm{m}$ region which is the minimum in optical absorption of silica-based optical fibers. Knowledge of the microscopic structures of the rare earth impurities in semiconductor hosts is very important for fabricating efficient injection-type light emitting devices. To understand the microscopic structures, the electron spin resonance (ESR) measurements, ${ }^{1-7}$ the extended x-ray absorption fine structure (EXAFS) measurements, ${ }^{8,9}$ and the Rutherford backscattering (RBS) channeling measurements ${ }^{10-13}$ have been performed on Er-doped GaAs, Er-doped Si, and Yb-doped InP.

ESR is a particularly good technique in understanding the atomic configurations of impurities in semiconductors, when various kinds of centers are simultaneously present in one sample. An isotropic ESR line for $\mathrm{Er}^{3+}\left(4 f^{11}\right)$ with cubic symmetry were observed in melt-grown GaAs crystals doped

\footnotetext{
${ }^{a}$ Electronic mail: ishiyama@mat.ims.tsukuba.ac.jp

b)Present address: Anritsu Corp., 1800 Onna, Atsugi, Kanagawa 243-5888, Japan.
}

with $\mathrm{Er}^{1}$ and GaAs implanted with $\mathrm{Er}^{2}$. However, the observed ESR line intensities suggested that the fraction of the cubic $\operatorname{Er}^{3+}\left(4 f^{11}\right)$ relative to the total number of $\mathrm{Er}$ atoms doped in these samples was small $(<0.1 \%)$. It was also suggested that the cubic $\mathrm{Er}^{3+}\left(4 f^{11}\right)$ does not exhibit PL spectrum.

It was shown that Er-doped GaAs grown with oxygen codoping by metal organic chemical vapor deposition (MOCVD) exhibits sharp and simple PL spectrum, when the GaAs host is photoexcited by photons with energy exceeding the band gap of GaAs. ${ }^{14}$ There are many kinds of Er centers having various different atomic configurations in the oxygencodoped GaAs layers. ${ }^{15}$ However, the analysis by PL and photoluminescence excitation (PLE) spectroscopy revealed that an Er center with a special atomic configuration (Er-2O) exhibits strong PL under host photoexcitation.

In this study, ESR measurements were performed on Erdoped GaAs samples grown with oxygen codoping by MOCVD. In addition to the isotropic line from the cubic center, other anisotropic ESR lines were newly observed in samples with oxygen codoping. We classified these ESR lines into typical four kinds of $\mathrm{Er}^{3+}\left(4 f^{11}\right)$ centers (A, B, C, and $\mathrm{D})$. The possible structures for these Er centers and the relation between these ESR centers and the luminescent $\mathrm{Er}$ center are discussed.

\section{EXPERIMENTAL PROCEDURES}

The samples used in this study were grown at $500{ }^{\circ} \mathrm{C}$ on (100)-oriented undoped GaAs substrates by MOCVD. 
Sources were triethyl gallium (TEG), arsine, and tris-isopropyl-cyclopentadienyl-Er. Oxygen codoping was performed in atmosphere containing $\mathrm{O}_{2}$ gas $(\leqslant 1 \mathrm{ppm})$. The thickness of the grown epitaxial layers were in the range of 2 to $3 \mu \mathrm{m}$. Depth profiles of $\mathrm{Er}$ and oxygen concentrations were measured by secondary-ion mass spectrometry (SIMS). The $\mathrm{Er}$ concentration ranged from $1.8 \times 10^{17}$ to 6.0 $\times 10^{18} \mathrm{~cm}^{-3}$ among the samples used here, and the Er impurities were almost uniformly distributed in the epitaxial layers. The oxygen concentrations in the samples with oxygen codoping were about two times higher than the Er concentrations. Oxygen was unintentionally incorporated into the epitaxial layer even without oxygen codoping, but the oxygen concentration in such a case were much lower than those in the samples with oxygen codoping.

The ESR measurements were performed at $4.2 \mathrm{~K}$ with an $X$-band $(\nu=9.05 \mathrm{GHz})$ spectrometer, using a cylindrical microwave cavity of $\mathrm{TE}_{011}$ mode. The sample was fixed with a TPFE rod in a quarts tube and was set in the center of the cavity. Consequently, misalignment of the sample is very slight with respect to the static magnetic field $H$. The static magnetic field $\mathrm{H}$ was swept from about 500 to $13500 \mathrm{G}$. The effective $g$ value $(=h \nu / \beta H)$ obtained in this $H$ range of the magnetic field is from about 0.5 to 16 . The relative ESR intensity was obtained by using an ESR line of $\mathrm{Mn}^{2+}$ in $\mathrm{MgO}$ measured simultaneously. The number of ESR-active Er atoms observed in the samples was evaluated using the ESR intensity of $\mathrm{Er}^{3+}$ in two standard samples of Er-doped $\mathrm{Y}_{3} \mathrm{Al}_{5} \mathrm{O}_{12}$ (YAG). Here, we assumed that all Er atoms in YAG are ESR active and the transition probabilities for ESR of $\mathrm{Er}^{3+}\left(4 f^{11}\right)$ are equal in GaAs and YAG. The ESR intensities for the two Er-doped YAG samples (Er concentration 0.005 and $0.05 \mathrm{wt} \%$ ) were proportional to the Er concentration. Since an Er atom occupies the yttrium site in YAG crystal, it would be reasonable to expect that all Er atoms in YAG crystals with these Er concentrations are ESR active. ${ }^{16}$ In order to investigate the relation between the observed ESR centers and the Er center showing the luminescence, the ESR measurements were performed under the illumination of a $\mathrm{He}-\mathrm{Ne}$ laser with a wavelength of $632 \mathrm{~nm}$. PLE measurements were also performed at $2 \mathrm{~K}$ using a multimode tunable $\mathrm{cw} \mathrm{NaCl}(\mathrm{OH})$ color laser as the excitation source.

\section{EXPERIMENTAL RESULTS AND DISCUSSION}

\section{A. ESR spectra for Er-oxygen complexes}

The isotropic ESR spectra of $\mathrm{Er}^{3+}\left(4 f^{11}\right)$ with a cubic symmetry are shown in Fig. 1. The spectrum (a) is for a sample with oxygen codoping $\left([\mathrm{Er}]=2.5 \times 10^{17} \mathrm{~cm}^{-3}\right.$, $[\mathrm{O}]=7.0 \times 10^{17} \mathrm{~cm}^{-3}$ ) and the spectrum (b) is for a sample without oxygen codoping $\left([\mathrm{Er}]=2.5 \times 10^{17} \mathrm{~cm}^{-3}, \quad[\mathrm{O}]\right.$ $=2.5 \times 10^{17} \mathrm{~cm}^{-3}$ ). The effective $g$ value of this resonance line is 5.95 . This is nearly equal to previously reported values of 5.921 in melt-grown GaAs crystals doped with $\mathrm{Er}^{1}$ and 5.947 in GaAs implanted with Er ions. ${ }^{2}$ The ESR intensity of this line in the sample with oxygen codoping decreases to a third of that in the sample without oxygen codoping.

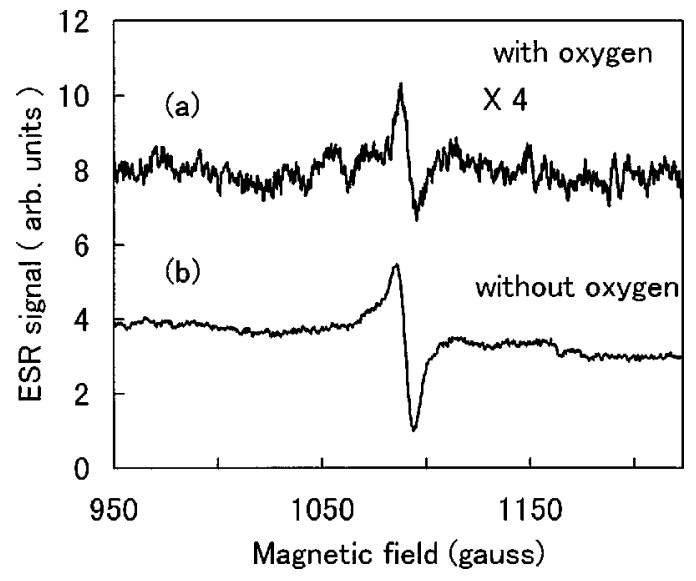

FIG. 1. Isotropic ESR lines originated from $\mathrm{Er}^{3+}\left(4 f^{11}\right)$ with a cubic symmetry for the samples with oxygen codoping (a) and without oxygen codoping (b). The measurements were performed at $4.2 \mathrm{~K}$ with a microwave frequency of $9.05 \mathrm{GHz}$. The microwave power was $0.8 \mathrm{~mW}$. The Er and oxygen concentrations are as follows: $[\mathrm{Er}]=2.5 \times 10^{17} \mathrm{~cm}^{-3}$, $[\mathrm{O}]=7.0 \times 10^{17} \mathrm{~cm}^{-3}$ for the sample with oxygen codoping (a), and $[\mathrm{Er}]=2.5 \times 10^{17} \mathrm{~cm}^{-3},[\mathrm{O}]=2.5 \times 10^{17} \mathrm{~cm}^{-3}$ for the sample without oxygen codoping (b).

In addition to this line, intense resonance lines for $\operatorname{Er}^{3+}\left(4 f^{11}\right)$ were observed in the samples with oxygen codoping, as shown in Fig. 2. The spectra (a) and (b) are for the sample with oxygen codoping and without oxygen
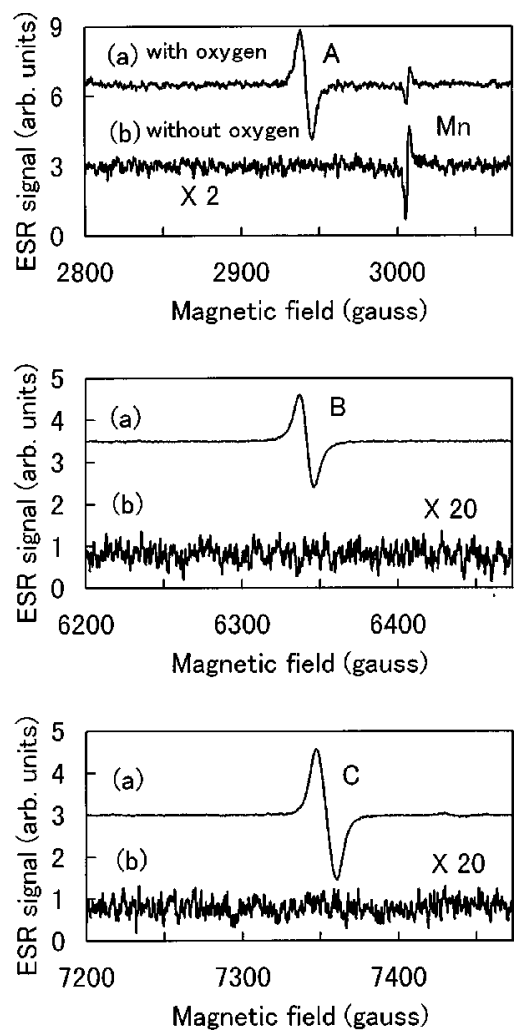

FIG. 2. Typical anisotropic ESR lines originated from three kinds of centers (A, B, and C) for the samples with oxygen codoping (a) and without oxygen codoping (b). An ESR line at about $3000 \mathrm{G}$ is from one of the hyperfine lines of $\mathrm{Mn}^{2+}$ in $\mathrm{MgO}$ measured at the same time. The samples were the same as those used in Fig. 1. The measurements were performed at $4.2 \mathrm{~K}$ and at $9.05 \mathrm{GHz}$. The microwave power was $1 \mu \mathrm{W}$ to avoid absorption saturation. The magnetic field was parallel to $\langle 110\rangle$ direction. 


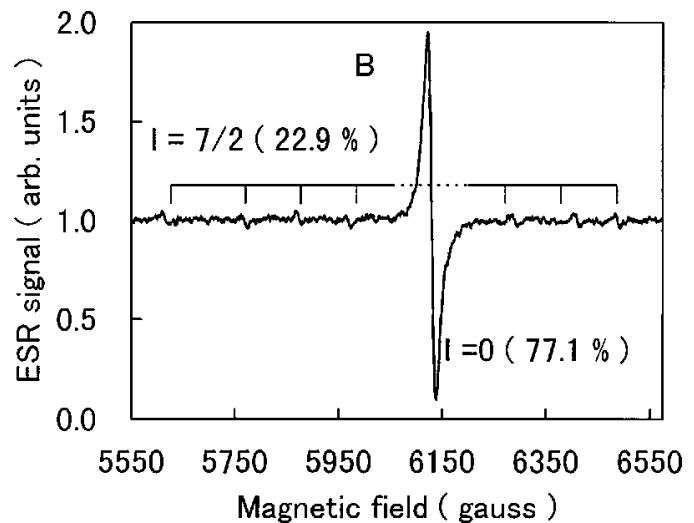

FIG. 3. Hyperfine lines due to ${ }^{167} \operatorname{Er}(I=7 / 2$, natural abundance $22.9 \%)$ for $\mathrm{B}$ center. The measurements were performed at $4.2 \mathrm{~K}$ and at $9.05 \mathrm{GHz}$. The microwave power was $5 \mu \mathrm{W}$. The magnetic field is tilted from [001] direction (the growth direction) by about $10^{\circ}$ in $(1 \overline{1} 0)$ plane.

codoping, respectively. These lines are anisotropic. The experimental results of the angular dependence and microwave power dependence show that they are originated from three kinds of $\mathrm{Er}^{3+}\left(4 f^{11}\right)$ centers. They are denoted $\mathrm{A}, \mathrm{B}$, and $\mathrm{C}$ centers, hereafter. These lines were not observed in the sample without oxygen codoping [the spectrum (b) in Fig. 2]. Therefore, the oxygen atoms neighbor these Er centers. The intensities of these lines are two orders of magnitude higher than that of the isotropic line with $g=5.95$. When the magnetic field is parallel to $\langle 11 \overline{0}\rangle$, the effective $g$ values are 2.19 for A center, 1.02 for $\mathrm{B}$ center, and 0.876 for $\mathrm{C}$ center. Besides the line due to ${ }^{168} \mathrm{Er}$ (nuclear spin of $I=0$ ), the hyperfine lines of B center due to ${ }^{167} \mathrm{Er}$ (nuclear spin of $I$ $=7 / 2$, natural abundance 22.9\%) are shown in Fig. 3. The ratio of intensities of these lines is in agreement with the ratio of their natural abundance. As well as B center, A and $\mathrm{C}$ centers also showed similar hyperfine lines due to ${ }^{167} \mathrm{Er}$.

As described above, only one signal with a cubic symmetry was observed in the sample without oxygen codoping. Comparing the ESR intensity of the isotropic center with that of $\mathrm{Er}^{3+}$ in Er-doped YAG, the number of Er atoms forming this cubic center was no more than $0.1 \%$ of all Er atoms in both samples with oxygen codoping and without oxygen codoping. This suggest that only a small fraction of Er atoms occupy either substitutional site or tetrahedral interstitial sites with cubic symmetry. Recently, it was shown by theoretical calculations that Er atom at Ga substitutional site is stable and that Er center coupled with other point defect are more stable than isolated Er atom in GaAs. ${ }^{17}$ This suggests that this isotropic signal with $g=5.95$ originates from a small fraction of isolated $\mathrm{Er}$ atoms (no more than $0.1 \%$ ) at $\mathrm{Ga}$ substitutional sites. Er centers coupled with other point defects have lower symmetry, so that they will show anisotropic ESR signals. The reasons why ESR signals from $\mathrm{Er}$ centers coupled with other point defects are not observed in the sample without oxygen-codoping are considered as follows:

(1) The wave function of $4 f$ electrons depends on the crystal field. Whether $\operatorname{Er}^{3+}\left(4 f^{11}\right)$ satisfy the ESR selection rule or not is determined by this wave function; i.e., the wave
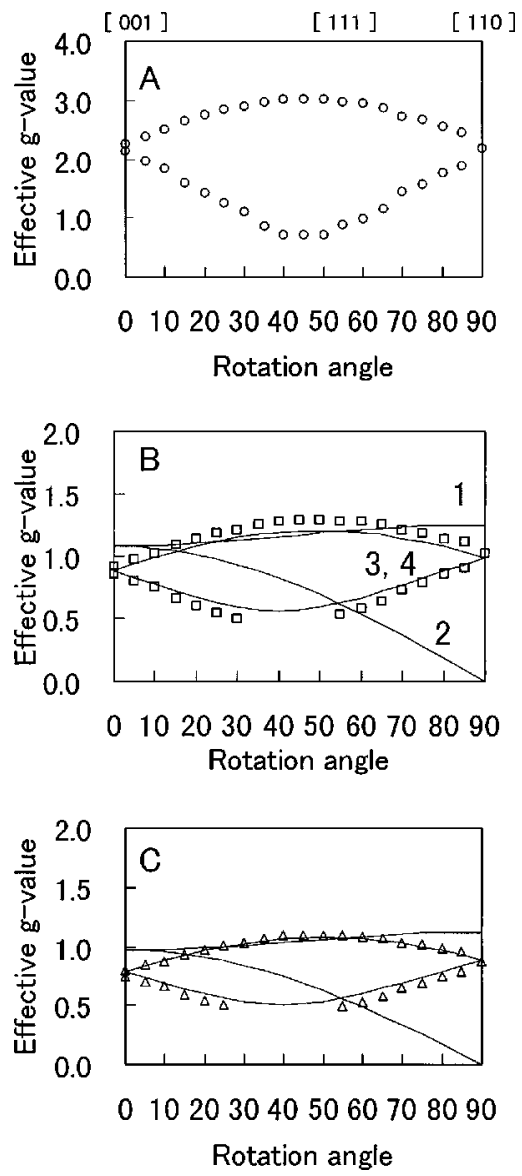

FIG. 4. Angular dependence of the ESR lines for the A (O), B ( $\square$ ), and C $(\triangle)$ centers. Calculated curves following to Eq. (1) are also shown for the B and $\mathrm{C}$ centers. The magnetic field is rotated in (1T̄0) plane from [001] direction (growth direction) to [110] direction.

function of the ground Kramers doublet should include $| \pm 1 / 2\rangle$ or $| \pm n / 2\rangle$ and $| \pm(n / 2 \pm 1)\rangle .^{18}$ Therefore, all Er centers in GaAs do not necessarily show ESR signals.

(2) If Er centers do not have well-defined structures (for example, Er atoms aggregates with various sizes), those centers will show very broad spectra which cannot be detected by ESR.

\section{B. Anisotropic $g$ tensors}

The ESR lines of the A, B, and C centers are dependent on the angle between the crystal direction and the magnetic field. Experimentally obtained angular dependence for the A, $\mathrm{B}$, and $\mathrm{C}$ centers are shown in Fig. 4. The magnetic field was rotated in the (110) plane from [001] (the direction of MOCVD growth, i.e., the growth direction) to [110]. The simulation was performed by using the effective spin Hamiltonian with the effective spin $S=1 / 2$, as follows:

$$
H=\mu_{B} \mathbf{H} \cdot \mathbf{g} \cdot \mathbf{S} .
$$

The calculated angular dependence for the B and C centers are shown in Fig. 4 by solid lines. The estimated principal $g$ values are $g_{B 1}=1.08, g_{B 2} \fallingdotseq 0.0$, and $g_{B 3}=1.25$ for B center, and $g_{C 1}=0.97, g_{C 2} \fallingdotseq 0.0$, and $g_{C 3}=1.12$ for $\mathrm{C}$ center. The $g_{1}$ axis (symmetry axis) is along a $\langle 100\rangle$ direction. The $g_{2}$ 
(a) configuration I

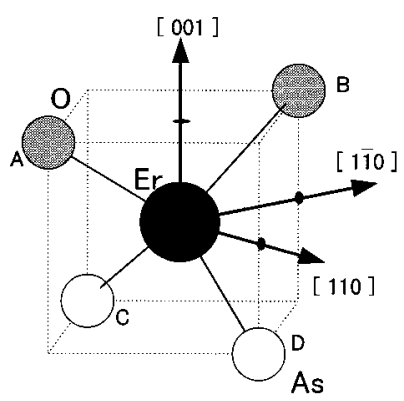

(b) configuration II

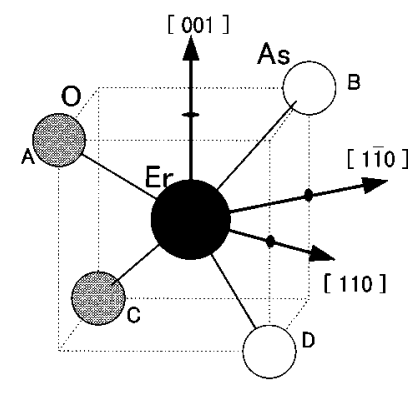

FIG. 5. Schematical Er-2O structure. The Er atom was assumed to occupy the Ga sublattice coordinated with two As atoms and two oxygen atoms. It is not clear whether two oxygen atoms are on substitutional site or interstitial sites.

and $g_{3}$ axes are along two $\langle 110\rangle$ directions in perpendicular to the $g_{1}$ axis. These principal $g$ values and axes indicate that the $\mathrm{B}$ and $\mathrm{C}$ centers have orthorhombic $C_{2 v}$ symmetry. The angular dependence of A center could not be explained by assuming the $C_{2 v}$ symmetry or symmetry higher than the $C_{2 v}$ symmetry. Therefore, it is suggested that A center has a symmetry lower than the $C_{2 v}$ symmetry.

The PL analysis ${ }^{19}$ indicated that the dominant luminescent Er center is coordinated with two As atoms and two oxygen atoms (Er-2O structure), as shown schematically in Fig. 5. Although the oxygen site is not known yet, we assumed here that the oxygen occupies the As lattice site. The $\mathrm{B}$ and $\mathrm{C}$ centers with the $C_{2 v}$ symmetry seem to have structures similar to the model of the luminescent center shown in Fig. 5. The $\mathrm{B}$ and $\mathrm{C}$ centers having the $C_{2 v}$ symmetry with symmetry axis along a $\langle 100\rangle$ direction have six equivalent configurations in the zinc-blende structure. The configurations with symmetry axis along [001] direction (the growth direction) are denoted by configuration I, as shown in Fig. 5(a). The configurations with symmetry axis along [100] or [010] direction perpendicular to the growth direction are denoted by configuration II, as shown in Fig. 5(b). The configuration I has two oxygen atoms occupying $\mathrm{A}$ and $\mathrm{B}$, or $\mathrm{C}$ and $\mathrm{D}$ in Fig. 5(a). The configuration II has two oxygen atoms occupying $\mathrm{A}$ and $\mathrm{C}$, or $\mathrm{B}$ and $\mathrm{D}$, or $\mathrm{A}$ and $\mathrm{D}$, or $\mathrm{B}$ and $\mathrm{C}$ in Fig. 5(b). When the magnetic field is rotated in the $\{110\}$ plane, the six equivalent configurations must show four ESR lines in general. In Fig. 4 where the magnetic field is rotated in (1 $\overline{1} 0)$ plane containing [001] direction (the growth direction), the configurations I and II are expected to show two set of ESR lines such as curves 1 and 2, and curves 3 and 4, respectively. So there should be four ESR lines in total. However, the B and C centers showed only two ESR lines each. The ESR lines observed for the B and C centers were from the configuration II with the principal $g_{1}$ axis along [100] and [010] directions perpendicular to the growth direction [001]; i.e., the ESR lines from the configuration I with the principal $g_{1}$ axis along the growth direction ([001] direction) were not observed. It is probable that the configuration II are preferentially formed, comparing with the configuration I. Although the magnetic field was also rotated in the (110) plane for the sample with Er concentration of 2.5 $\times 10^{17} \mathrm{~cm}^{-3}$, the same result was obtained.

Optical anisotropy of the dominant Er center was already studied by measuring luminescence polarization. ${ }^{20}$ It was reported that the luminescence intensity shows difference in detecting light with polarization parallel to [110] and [1 $\overline{1} 0]$, but it is the same parallel to [100] and [010]. Therefore, the optical anisotropy results suggest that two oxygen atoms preferentially occupied either $\mathrm{A}$ and $\mathrm{B}$, or $\mathrm{C}$ and $\mathrm{D}$ of the configuration I. However, ESR results suggest that no significant centers with configuration I are produced. This is not consistent with the anisotropic host-excited photoluminescence. There are two possibilities for this inconsistency. First, Er centers observed by ESR may be different from those observed by the anisotropic host-excited photoluminescence. Second, although Er centers observed by ESR are the same as those observed by the anisotropic host-excited photoluminescence, there may be other reasons that are not known yet.

The principal axes of the $\mathrm{B}$ and $\mathrm{C}$ centers are along the same directions, whereas the principal $g$ values for these centers are different. These suggest that the $\mathrm{B}$ and $\mathrm{C}$ centers have the same configurations for the nearest-neighbor sites and different configurations for the second-nearest-neighbor sites. The $4 f$ shell of Er is shielded by the filled $5 s$ and $5 p$ shells. The crystal field which influences the $4 f$ shell is dominated by the nearest-neighbor atoms. However, a small contribution of the second-nearest-neighbor atoms to the crystal field could cause the difference principal $g$ values, as seen for the $\mathrm{B}$ and $\mathrm{C}$ centers. We speculate that the nearestneighbors configuration for A center having a lower symmetry is different from those for the $\mathrm{B}$ and $\mathrm{C}$ centers.

\section{D center}

In addition to the ESR lines for the $\mathrm{A}, \mathrm{B}$, and $\mathrm{C}$ centers, the lines for another $\mathrm{Er}^{3+}\left(4 f^{11}\right)$ center (D center) were observed in the samples with oxygen codoping, as shown in Fig. 6. The spectra (a) and (b) are for the samples with oxygen codoping having different Er concentration. These lines were anisotropic and observed clearly in the samples with the Er concentration above $10^{18} \mathrm{~cm}^{-3}$. The ESR intensities of these lines for $\mathrm{D}$ center were about one order of magnitude lower than those for the A, B, and C centers. These lines are classified into strong lines (D-1) and weak lines (D-2). The angular dependence for the D-1 and D-2 are shown in Fig. 7. The magnetic field was rotated in the $(1 \overline{1} 0)$ plane from [001] (the growth direction) to [110]. The simulation was done by using Eq. (1). The D-1 and D-2 are of the $\langle 110\rangle$-axial symmetry. The principal $g$ values are $g_{\mathrm{D}-1_{\|}}$ $=15.4, g_{\mathrm{D}-1_{\perp}}=0.8, \phi= \pm 1^{\circ}$ for the D-1, and $g_{\mathrm{D}-2 \|}=16.4$, $g_{\mathrm{D}-2 \perp}=2.8, \phi= \pm 1^{\circ}$ for the D-2. The $g_{\|}$axis is tilted from the $\langle 110\rangle$ direction in $\{100\}$ plane by the angle $\phi$. Because these lines were not observed in the sample without oxygen codoping, oxygen atoms neighbor to the Er atom in the D center, as are the case in $\mathrm{A}, \mathrm{B}$, and $\mathrm{C}$ centers. The structure for $\mathrm{D}$ center with the $\langle 110\rangle$-axial symmetry is different from the structure shown in Fig. 5. 


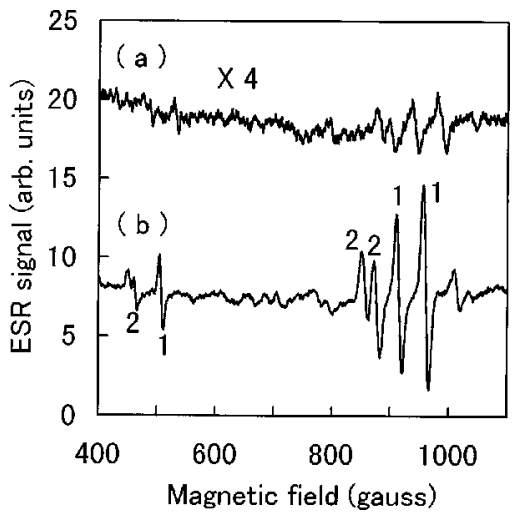

FIG. 6. Anisotropic ESR lines originated from D center in the samples with oxygen codoping. The Er and oxygen concentrations in the samples are as follows; $[\mathrm{Er}]=2.5 \times 10^{17} \mathrm{~cm}^{-3}, \quad[\mathrm{O}]=7.0 \times 10^{17} \mathrm{~cm}^{-3}$ for (a) and $[\mathrm{Er}]=1.4 \times 10^{18} \mathrm{~cm}^{-3},[\mathrm{O}]=4.5 \times 10^{18} \mathrm{~cm}^{-3}$ for $(\mathrm{b})$. The measurements were performed at $4.2 \mathrm{~K}$ and at $9.05 \mathrm{GHz}$. The microwave power was $0.8 \mathrm{~mW}$. The magnetic field is intended to be set parallel to $\langle 110\rangle$ direction. A little difference between directions of magnetic field in (a) and (b) causes different position of ESR lines between ESR spectra (a) and (b). These lines are classified into strong lines (D-1) and weak lines (D-2).

It was reported that ESR lines from $\mathrm{Er}^{3+}$ center with $\mathrm{Er}_{2} \mathrm{O}_{3}$ structure were observed in a $\mathrm{Si}$ wafer implanted with Er and oxygen. ${ }^{4}$ The ESR lines for the D center are observed in the same region of the magnetic field as the ESR lines observed in $\mathrm{Si}$. It is known that $\mathrm{Er}^{3+}$ ion occupies two different sites in $\mathrm{Er}_{2} \mathrm{O}_{3}$. One is trigonal $C_{3 i}$ site and the other is orthorhombic $C_{2 v}$ site. The principal $g$ values for $\mathrm{Er}^{3+}$ in $\mathrm{Y}_{2} \mathrm{O}_{3}$ which has the same crystal structure as $\mathrm{Er}_{2} \mathrm{O}_{3}$ are $g_{\|}$ $=12.176, g_{\perp}=3.319$ for trigonal $C_{3 i}$ site, and $g_{x}=1.645$, $g_{y}=4.892$, and $g_{z}=12.314$ for an orthorhombic $C_{2 v}$ site. ${ }^{21}$ These $g$ values are not comparable to those for D-1 and D-2 centers. This suggests that the structure of $\mathrm{D}$ center is entirely different from $\mathrm{Er}_{2} \mathrm{O}_{3}$ structure.

Although we do not have definite models for D-1 and D-2 centers at present, they have the same symmetry, whereas the $g$ values for those centers are different. It is likely that the atomic configurations of the second-nearest neighbor sites for the D-1 and D-2 centers are different, as are the cases in $\mathrm{B}$ and $\mathrm{C}$ centers.
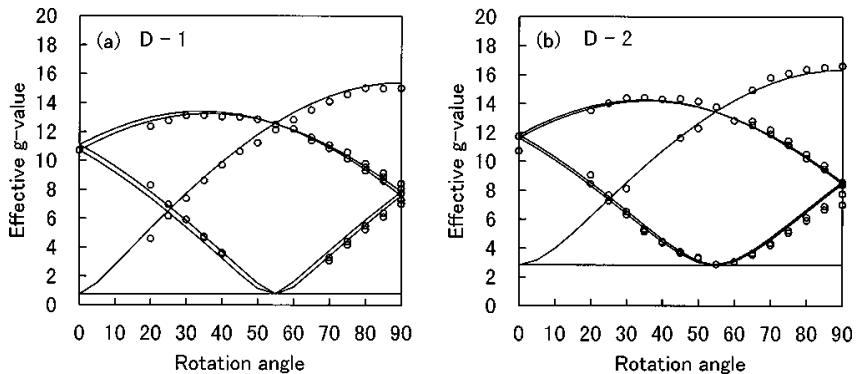

FIG. 7. Angular dependence of the ESR lines for the D-1 and D-2. Calculated curves following to Eq. (1) are also shown by solid lines. The magnetic field is rotated in (1 $1 \overline{1} 0)$ plane from [001] direction (the growth direction) to [110] direction.
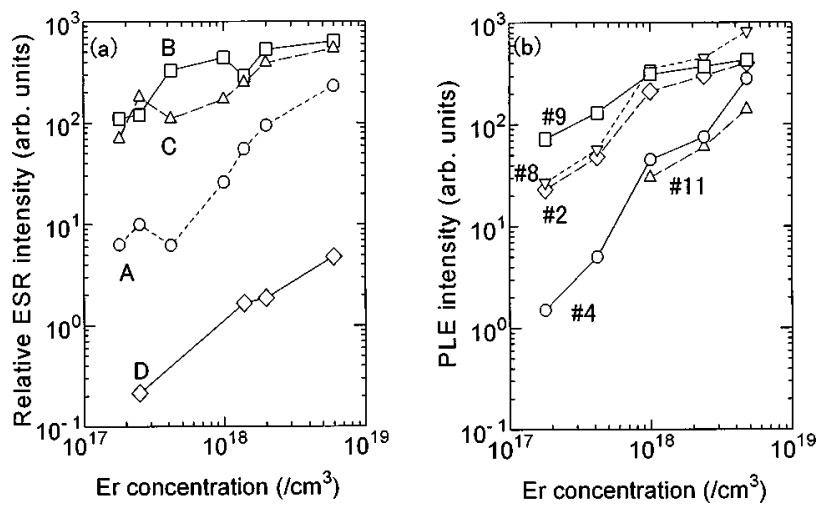

FIG. 8. ESR signal intensity for A $(\bigcirc)$, B $(\square), \mathrm{C}(\triangle)$, and D $(\diamond)$ centers (a) and PLE intensity for Nos. $2(\diamond), 4(\bigcirc), 8(\nabla), 9(\square)$, and $11(\triangle)$ lines $(b)$, as a function of Er concentration. The ESR intensity was normalized by the volume of the epitaxial layer.

\section{Relation between ESR centers and luminescent center}

The Er concentration dependence of ESR and PLE intensities are shown in Figs. 8(a) and 8(b), respectively. The ESR intensity was normalized by the volume of the epitaxial layer. The total number of Er atoms forming A, B, C, D and $g=5.95$ (cubic) centers was estimated to be approximately 90\% of all Er atoms in the samples when Er concentrations are lower than $[\mathrm{Er}]=4.8 \times 10^{17} \mathrm{~cm}^{-3}$. In this estimation, we assumed that the transition probabilities for ESR are equal for all Er centers in GaAs and YAG. The reasons of this assumption is as follows: The microwave magnetic field is perpendicular to the static magnetic field $\mathrm{H}$. The transition probability for ESR depends on a component, i.e., $g_{[1 \overline{1} 0]}$, of the principal $g$ values $\left(g_{1}, g_{2}\right.$, and $\left.g_{3}\right)$, perpendicular to the static field and on $\left\langle M-1\left|J_{-}\right| M\right\rangle \cdot{ }^{18}$ However, the experimental $g$ values obtained by using the effective spin Hamiltonian with the effective spin $\widetilde{S}=1 / 2$ [Eq. (1)] are different from the principal $g$ values $\left(g_{1}, g_{2}, g_{3}\right)$, except for directions of their axis. This is caused by a fact that the wave functions are not known since they will be complicated linear combination of states $\left|J_{z}=1 / 2\right\rangle,\left|J_{z}=3 / 2\right\rangle, \cdots,\left|J_{z}=13 / 2\right\rangle$, and $\left|J_{z}=15 / 2\right\rangle$ for the Kramers doublet of $\operatorname{Er}^{3+}\left(4 f^{11}\right)$. Thus, the term of $\left(g_{1}, g_{2}\right.$, and $\left.g_{3}\right)$ and $\left\langle M-1\left|J_{-}\right| M\right\rangle$ cannot be estimated. The wave functions will be various for all the Er centers in GaAs and YAG observed by ESR. Therefore, we assumed that the transition probabilities for ESR are equal for all the Er centers in GaAs and YAG, although the experimental $g_{[1 \overline{1} 0]}$ values for A, B, C, and D centers were various.

PLE lines originated from many kinds of Er centers have been observed in Er-doped GaAs with oxygen codoping. ${ }^{22}$ The number of the main PLE lines is approximately 11, and the PLE lines were numbered from No. 1 to 11 in Ref. 22. The intensities of the typical PLE centers among these lines are shown in Fig. 8(b). The PLE line No. $9(0.8058 \mathrm{eV})$ indicates a center exhibiting PL under host photoexcitation, because the identical PL spectrum is observed when PLE line No. 9 is excited.

The Er concentration dependence of ESR and PLE intensities can be classified into two classes. One is the dependence that the intensities increase with increasing Er concen- 

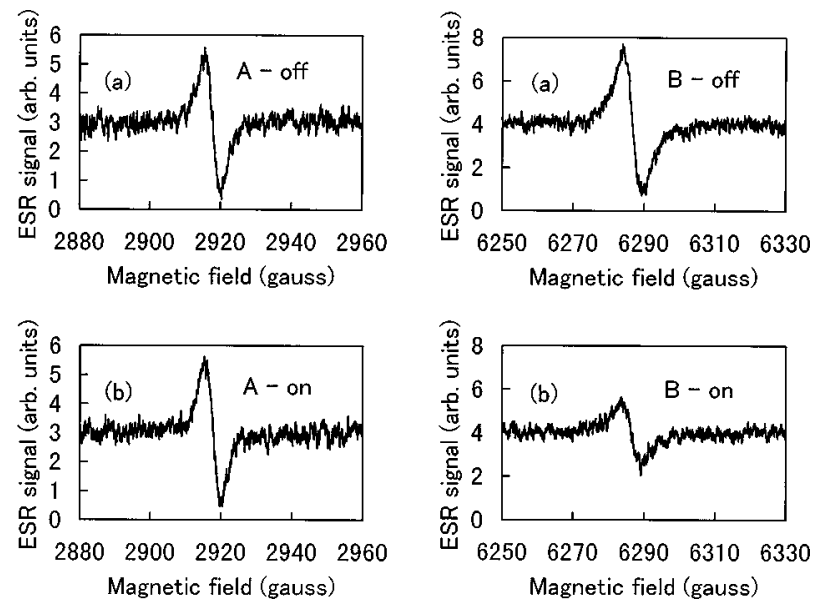

FIG. 9. ESR signals for the A and B centers under no illumination (a) and under illumination (b). The samples were irradiated with a $\mathrm{He}-\mathrm{Ne}$ laser light $(632 \mathrm{~nm})$ at $4.2 \mathrm{~K}$. The Er and oxygen concentrations in the sample with oxygen codoping are $[\mathrm{Er}]=2.5 \times 10^{17} \mathrm{~cm}^{-3},[\mathrm{O}]=7.0 \times 10^{17} \mathrm{~cm}^{-3}$. The ESR intensity of $\mathrm{B}$ center decreased under illumination, while that of $\mathrm{A}$ center showed no changes in the intensities.

tration, the other is the dependence that the intensities are saturated above $[\mathrm{Er}] \geqslant 10^{18} \mathrm{~cm}^{-3}$. The A and D centers observed in the ESR measurements and Nos. $4(0.8045 \mathrm{eV})$ and $11(0.8065 \mathrm{eV})$ centers observed in PLE measurements belong to the former. The $\mathrm{B}$ and $\mathrm{C}$ centers of the ESR and the Nos. $2(0.8039 \mathrm{eV}), 8(0.8057 \mathrm{eV})$, and 9 centers of the PLE belong to the latter. From these classifications, it is probable that the luminescent Er center (PLE line No. 9) corresponds to either $\mathrm{B}$ or $\mathrm{C}$ center.

The changes in ESR intensities for the A and B centers under the illumination with $632 \mathrm{~nm}$ laser light are shown in Fig. 9. The total area of the sample was illuminated by $\mathrm{He}-\mathrm{Ne}$ laser. The power is about $2 \mathrm{~mW} / \mathrm{cm}^{2}$. The ESR intensity of $\mathrm{B}$ center decreased under the illumination by about $30 \%$, while the A, C, and D showed no changes in their intensities. A possible explanation of this experimental result is that the $4 f$ shell of only $\mathrm{B}$ center is excited under host photoexcitation, while those of the $\mathrm{A}, \mathrm{C}$, and $\mathrm{D}$ center are not excited. Although the angular dependence of $B$ and $C$ centers is not consistent with the anisotropic host-excited photoluminescence, as described in Sec. III B, we think that there should be unknown reasons for the inconsistency. Consequently, the Er concentration dependence of ESR and PLE intensities and the ESR measurements under illumination are thought to suggest that the luminescent Er center (PLE line No. 9) corresponds to B center with $C_{2 v}$ symmetry having a $\mathrm{Er}-2 \mathrm{O}$ structure.

\section{CONCLUSIONS}

The ESR measurements were performed on Er-doped GaAs grown with oxygen codoping by MOCVD. The isotropic ESR line from $\mathrm{Er}^{3+}\left(4 f^{11}\right)$ with a cubic symmetry was observed in the samples with oxygen codoping and without oxygen codoping. In addition to this line, the anisotropic ESR lines from four kinds of $\operatorname{Er}^{3+}\left(4 f^{11}\right)$ centers $(\mathrm{A}, \mathrm{B}, \mathrm{C}$, and $D)$ were newly observed in the samples with oxygen codoping. The $\mathrm{B}$ and $\mathrm{C}$ centers is of orthorhombic $C_{2 v}$ symmetry and A center has a lower symmetry. The $\mathrm{D}$ center has the $C_{3 i}$ symmetry. It is suggested that B center with a Er-2O structure corresponds to the luminescent Er center.

${ }^{1}$ M. Baeumler, J. Schneider, F. Kohl, and E. Tomzing, J. Phys. C 20, L963 (1987)

${ }^{2}$ P. B. Klein, F. G. Moon, and H. B. Dietrich, Appl. Phys. Lett. 58, 502 (1991).

${ }^{3}$ T. Ishiyama, E. Katayama, K. Takahei, A. Taguchi, and K. Murakami, Mater. Sci. Forum 258-263, 1559 (1997).

${ }^{4}$ J. D. Carey, J. F. Donegane, R. C. Barklie, F. Prioro, G. Franzo, and S. Coffa, Appl. Phys. Lett. 69, 3845 (1996).

${ }^{5}$ V. F. Masterov, V. V. Romanov, and K. F. Shtel'makh, Sov. Phys. Solid State 25, 824 (1983).

${ }^{6}$ B. Lambert, Y. Toudic, G. Grandpierre, A. Rupert, and A. Le Corre, Electron. Lett. 24, 1446 (1988).

${ }^{7}$ T. Ishiyama, K. Murakami, K. Takahei, and A. Taguchi, J. Appl. Phys. 82, 4457 (1997).

${ }^{8}$ D. L. Adler, D. C. Jacobson, D. J. Eaglesham, M. A. Marcus, J. L. Benton, J. M. Poate, and P. H. Citrin, Appl. Phys. Lett. 61, 2181 (1992).

${ }^{9}$ A. Terrasi, G. Franzo, S. Coffa, F. Priolo, F. Dacapito, and S. Mobilio, Appl. Phys. Lett. 70, 1712 (1997).

${ }^{10}$ J. Nakata, M. Taniguchi, and K. Takahei, Appl. Phys. Lett. 61, 2665 (1992).

${ }^{11}$ Y. S. Tang, Z. Jingping, K. C. Haesman, and B. J. Sealy, Solid State Commun. 72, 991 (1989).

${ }^{12}$ A. Kozanecki, R. Wilson, B. J. Sealy, J. Kaczanowski, and L. Nowicki, Appl. Phys. Lett. 67, 1847 (1995).

${ }^{13}$ U. Wahi, A. Vantomme, J. De Wachter, R. Moons, G. Langouche, J. G. Marques, J. G. Correia, and ISOLDE Collaboration, Phys. Rev. Lett. 79, 2069 (1997).

${ }^{14}$ K. Takahei and A. Taguchi, J. Appl. Phys. 74, 1979 (1993).

${ }^{15}$ K. Takahei and A. Taguchi, J. Appl. Phys. 77, 1735 (1995).

${ }^{16}$ P. G. Baranov, V. I. Zhekev, T. M. Murina, A. M. Prokhorov, and V. A. Khramtsov, Sov. Phys. Solid State 29, 723 (1987).

${ }^{17}$ A. Taguchi and T. Ohno, Phys. Rev. B 56, 9477 (1997).

${ }^{18}$ A. Abragam and B. Bleaney, Electron Paramagnetic Resonance of Transition Ions (Clarendon, Oxford, 1970).

${ }^{19}$ K. Takahei, A. Taguchi, Y. Horikoshi, and J. Nakata, J. Appl. Phys. 76, 4332 (1994)

${ }^{20}$ R. A. Hogg, K. Takahei, A. Taguchi, and Y. Horikoshi, Appl. Phys. Lett. 68, 3317 (1996)

${ }^{21}$ G. Schafer and S. Scheller, Phys. kondens. Mater. 5, 48 (1966).

${ }^{22}$ K. Takahei, A. Taguchi, and R. A. Hogg, J. Appl. Phys. 82, 3997 (1997). 